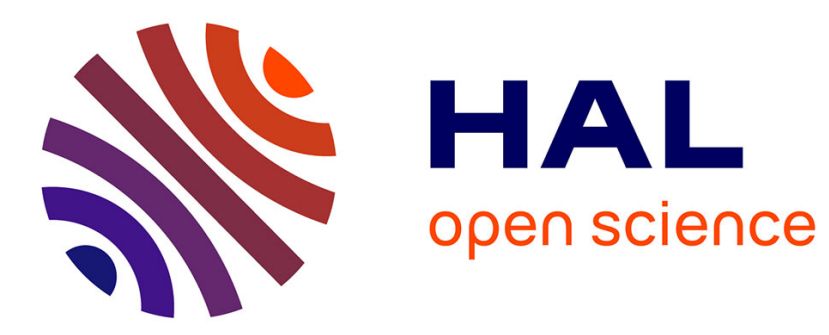

\title{
Les réformes de l'enseignement comme révélateur et amplificateur des fractures enseignantes
}

\author{
Farinaz Fassa-Recrosio, Pierre Bataille
}

\section{To cite this version:}

Farinaz Fassa-Recrosio, Pierre Bataille. Les réformes de l'enseignement comme révélateur et amplificateur des fractures enseignantes. Education et Sociétés: Revue internationale de sociologie de l'éducation, 2019, nº3 (1), pp.5. 10.3917/es.043.0005 . hal-02371874

\section{HAL Id: hal-02371874 \\ https://hal.science/hal-02371874}

Submitted on 20 Nov 2019

HAL is a multi-disciplinary open access archive for the deposit and dissemination of scientific research documents, whether they are published or not. The documents may come from teaching and research institutions in France or abroad, or from public or private research centers.
L'archive ouverte pluridisciplinaire HAL, est destinée au dépôt et à la diffusion de documents scientifiques de niveau recherche, publiés ou non, émanant des établissements d'enseignement et de recherche français ou étrangers, des laboratoires publics ou privés. 


\title{
Les réformes de l'enseignement comme révélateur et amplificateur des fractures enseignantes
}

\author{
Farinaz Fassa (1) et Pierre Bataille (2) ${ }^{1}$
}

\begin{abstract}
Résumé
Dans une conjoncture marquée par les politiques d'austérité, la recherche d'efficacité, la volonté d'éviter l'exclusion ou d'intégrer la diversité, les systèmes d'éducation et de formation se diversifient et se complexifient, suscitant des réformes inspirées de la Nouvelle gestion publique (NGP). Prenant acte de ce nouveau cadrage, ce dossier examine les façons dont ces réformes ont transformé les conditions d'exercice des métiers de l'éducation. Il met l'accent sur l'hybridité de ces processus, insiste sur la nécessité de prendre en compte les situations spécifiques pour comprendre ce que font les principes de la NGP aux fractions des professionnels de terrain en éducation dans l'espace francophone (France, Belgique, Québec et Suisse romande). Tous et toutes ne gagnent pas également à l'adoption de ces réformes, leur appropriation tenant aussi aux caractéristiques sociales des diverses fractions dont sont constitués les corps enseignants. Offrir des éléments éclairant les raisons pour lesquelles les professionnels adhèrent, acceptent ou récusent des réformes qui bouleversent les définitions antérieures de la professionnalité des enseignants et analyser les usages qu'ils et elles font de ces réformes permet aussi de dessiner la carte des territoires professionnels. Ceux-ci se rétrécissent ou prennent de l'ampleur lorsqu'il s'agit à la fois de rendre des comptes et d'endosser la responsabilité de réaliser des objectifs fixés par les politiques comme partie intégrante de l'activité professionnelle.
\end{abstract}

Ref. originale : Fassa F. et Bataille P. (2019), "Les réformes de l'enseignement comme révélateur et amplificateur des fractures enseignantes", Éducation et Sociétés, 43, pp.5-23

\section{Introduction}

Dans une conjoncture marquée par les politiques d'austérité, la recherche d'efficacité, la volonté d'éviter l'exclusion ou d'intégrer la diversité, les systèmes d'éducation et de formation se diversifient et se complexifient (Maroy 2006), suscitant nombre de réformes. Elles touchent autant les processus de production des politiques éducatives que les curricula des différents degrés des systèmes d'éducation/formation (SEF), les objectifs pédagogiques ou la définition des professionnalités dans le champ éducatif. Ces réformes sont modulées, selon les secteurs et les segments professionnels des services publics, par l'adoption de politiques de gestion visant un accroissement de l'efficience, la

${ }^{1}$ (1) Observatoire de l'éducation et de la formation (OBSEF) et Centre en études genre (CEG), Université de Lausanne; (2) Laboratoire de Recherche sur les Apprentissages en Contexte (LaRAC) et Observatoire de l'éducation et de la formation (OBSEF), Université Grenoble Alpes

Auteur correspondant:

Farinaz Fassa, Université de Lausanne. transparence des processus et de la mise en œuvre. Ces changements, dus à l'adoption des principes de Nouvelle Gestion Publique (NGP), sont observables à l'échelle internationale, mais ils se déclinent différemment selon les situations nationales et les formulations des visées et missions de l'éducation et de la formation par les politiques éducatives (Demazière, Lessard \& Morrissette 2013). En ce qui concerne les professions éducatives, une pluralité des modèles nationaux subsiste indéniablement au-delà des points communs liés à la relative crise d'attractivité de ces métiers.

$\mathrm{Si}$ la NGP touche toutes les administrations publiques et sert de soubassement aux réformes entreprises (Bezes et al. 2011, Emery \& Giauque 2014), elle marque spécifiquement les personnels des différents secteurs du service public, voire les divers segments d'un même corps de métier. Ce dossier tente de préciser les conséquences de ces changements sur les personnels éducatifs, notamment les conceptions de la professionnalité et les identités professionnelles. Ces aspects, relativement peu étudiés, tant les bouleversements en cours sont d'ampleur, ont suscité des réflexions globales. A ceci, s'ajoute 
l'environnement technologique (dont l'usage généralisé des outils numériques) qui a participé à dévaloriser les savoirs disciplinaires dont les formateurs avaient le monopole mais aussi celui qui a fait parfois décrire les enseignants et leur formation comme responsables de l'échec des politiques de démocratisation (Dubet 2014).

Plusieurs décennies après que les modèles anglosaxons en ont dessiné la silhouette, la multiplicité des formes de la NGP est telle que Demazières, Lessard et Morrissette $(2013,7)$ parlent d'un "tableau [...] baroque" lorsqu'il s'agit de dessiner ses effets sur le travail dans le secteur public. Ce dossier d'Éducation et Sociétés-32 (2013) brosse un panorama nuancé et riche des conséquences de la NGP dans les métiers de relation en France, au Québec et en Belgique. Les recherches présentées dans le domaine de l'éducation et de la formation insistent toutefois surtout sur l'action des hiérarchies intermédiaires et sur la situation paradoxale dans laquelle elles se trouvent.

Pour compléter ce tableau, le présent dossier, centré sur les acteurs et actrices en prise directe avec les usagers et usagères, s'ancre dans une lecture prenant la professionnalité comme entrée : l'adoption de cet angle a paru adéquat pour poursuivre l'entreprise de ces chercheurs et répondre à leur vœu de développer des travaux susceptibles d'éclairer les "conséquences des différentes modalités de mise en œuvre en termes de multiplication des lignes de partage au sein des groupes professionnels, de recomposition de ces groupes, de déplacements de leurs périmètres" (Demazières, Lessard \& Morrissette 2013, 14). Les articles de ce dossier ont en commun d'offrir des éléments éclairant les raisons pour lesquelles les professionnels adhèrent, acceptent ou récusent des réformes qui bouleversent les définitions antérieures de la professionnalité des enseignants. Dans une conjoncture décrite comme "hybride" par Emery et Giauque (2014) -car les organisations publiques doivent composer entre un alignement sur les impératifs de la performance et le maintien de services favorisant les processus démocratiques, approcher les effets des réformes inspirées par la NGP et les usages qu'en font les professionnels permet aussi de saisir les territoires professionnels qui se rétrécissent ou prennent de l'ampleur dans les mondes de l'école et de la formation.

\section{À l'école de la Nouvelle gestion publique}

\section{Des processus ambivalents}

Les interprétations divergent quant aux processus décrits sous le terme générique de nouvelle gestion publique, mais elles se rejoignent sur le fait que les principes de la NGP sous-tendent aujourd'hui les politiques des États dans tous les secteurs du service public. En cours depuis plus de quatre décennies en Grande-Bretagne et aux États-Unis, la réorganisation actuelle des systèmes administratifs les dote d'instruments et de méthodes de management qui étaient l'apanage des entreprises du secteur privé. Si la culture des résultats, la mesure de la performance, la contractualisation et la redevabilité (ou reddition de compte) en sont les traits les plus marquants, elles doivent composer avec les anciennes manières de penser la gestion publique pour proposer des réformes fondées d'une part sur la réalisation d'objectifs, l'évaluation des performances des organisations et de leurs professionnels et, d'autre part, sur la prise en compte plus largedes demandes des usagers (Demazières, Lessard \& Morissette 2013).

De telles transformations ont pris une acuité particulière dans les champs de l'éducation et de la formation, du fait notamment des fonctions de socialisation, d'intégration et de distribution (Dubet 2009) que jouent ces secteurs. Les remaniements engagés par l'adoption de NGP y ont d'autant plus manifesté du caractère hybride (Emery et Giauque 2014) que leur conjoncture internationale a été marquée par un discours décrivant les pays industrialisés comme des économies de la connaissance alors que les indices de chômage étaient très élevés chez les jeunes dans plusieurs pays. L'alliance faite prioritairement entre bien-être économique des nations et action éducative et de formation a rendu le débat qu'ont initié promoteurs et détracteurs de la NGP (Laval 2003, Bruno, Clément \& Laval 2010, Laval et al. 2011) très vif dans le monde de l'école et de la formation. Les prises de positions tranchées ont été d'autant plus nombreuses "dans des échanges qui mêlent, sans rigueur, arguments scientifiques et 'profanes', faits et normes voire, en dernier ressort, interpellation du 'bon sens"' (Mons 2008a, 5) que les appels à la mise en œuvre de la NGP en éducation ont pris place dans un environnement scientifique et médiatique recourant systématiquement aux données probantes pour organiser et gérer l'action publique 
(evidence-based policies). Les débats se sont alors polarisés sur les résultats des élèves et des étudiants et sur les facteurs qui y contribuent, des études ayant montré que l'absence de diplôme reconnu était un risque accru de chômage (CE 2012, Poullaouec 2010). Les conséquences de la massification des études supérieures ont aussi été discutées dans les médias (cf. la polémique entre Tristan Poullaouec d'un côté et François Dubet et Marie Duru-Bellat de l'autre sur l'emprise des diplômes - Dubet, Duru-Bellat \& Poullaouec, 2006). Si des travaux (notamment Mons 2008b, Baudelot \& Establet 2009, Duru-Bellat, Dubet \& Vérétout 2011) ont nuancé les réflexions quant aux bénéfices que pouvaient offrir les comparaisons internationales, l'importance du capital scolaire est apparue aux yeux du public comme le plus important facteur d'intégration sociale ultérieure . Or, il est dorénavant un acteur inévitable de politiques publiques pensées comme redevables.

\section{Performance, évaluation et redevabilité}

Le courant de recherche de sur l'efficacité de l'école ( « school effectiveness »), apparu dans la littérature anglo-saxonne des années 1980, a essaimé dans la plupart des espaces académiques nationaux. Il a même inspiré certaines politiques publiques dans le sens d'une "obligation de résultat intelligente" (Normand \& Derouet 2011). En faisant porter l'accent sur les apprenants et apprenantes ou les bénéficiaires du travail social et leurs apprentissages (Lang 2001), le changement de paradigme induit par ce courant de recherche est un bouleversement majeur pour les professions éducatives (Cattonar 2001, Maroy 2006, 2008). Les comparaisons internationales mettent en évidence l'action des enseignants et des établissements sur les performances des élèves, une centralité plus grande donnée aux dimensions locales et aux établissements (Bressoux 2009, Duru-Bellat 2011, Félouzis \& Charmillot 2012), les acteurs professionnels de l'éducation se multipliant et devant se coordonner pour répondre à des besoins spécifiques.

Cette dévolution de responsabilité à l'échelle locale s'est accompagnée d'une reconfiguration de la normativité scolaire (Maroy 2018). Répondant à l'impératif de réarticuler les "finalités de justice (ellesmêmes redéfinies en termes d'équité et d'inclusion) avec des finalités plus managériales d'efficacité" (Maroy 2018, 287), les réformes les plus récentes mettraient au cœur des transformations l'alliance d'une école efficace et inclusive, redéfinissant ainsi la place de tous les acteurs de l'éducation formelle. Dans un tel paradigme, privilégiant la réussite de tous et ne tolérant aucun échec, les parents se sentent en droit d'attendre de tous les professionnels de l'éducation qu'une attention soit portée à leurs enfants par des pédagogies et dispositifs adaptés. Selon Barrère (2013), le recours croissant à la notion de dispositif rend compte de ces métamorphoses. Articulant pérennité de la forme scolaire et impératifs de réformes, il évite une mise en cause des structures et de leur adéquation aux missions éducatives telles qu'elles se déclinent actuellement. . Il semble aussi apte à répondre aux demandes des parents d'élèves, car il relie les situations connues de la forme scolaire à des innovations accroissant la redevabilité dont doivent faire preuve les personnels éducatifs.

Le développement des études focalisées sur la question de l'efficacité de l'école ont contribué dans les faits autant à la transformation des visées que des processus d'évaluation des politiques éducatives, la pensée s'appuyant désormais sur une analyse comparative (《 benchmarking ») proposée et vulgarisée par les résultats des enquêtes internationales telles PISA, qui accroissent les demandes de redditions de comptes des publics concernés. La logique d'évaluation et de performance ne s'arrête toutefois pas aux évaluations internationales qui permettraient selon leurs promoteurs de "se fixer des objectifs d'amélioration, par exemple atteindre les scores moyens d'autres pays ou parvenir à un degré d'équité plus élevé en termes de perspectives et de résultats éducatifs; comprendre les points forts et les points faibles de leur système d'éducation" (OCDE 2004, 21). Elle descend à l'échelon local, voire à celui des établissements et/ou des professionnels, et peut devenir un puissant levier de responsabilisation de certaines fractions des enseignants. Mais cette dévolution de responsabilité, comme l'adhésion à cette nouvelle posture, se produit en raison d'environnements étroits qui conduisent à des "découplages" (Bezes et al. 2011) plus ou moins marqués selon les modalités des interactions entre les divers acteurs professionnels et les histoires, très situées, des établissements (cf. Buisson-Fenet et Pons ci-après).

Cette seule logique des résultats va à l'encontre d'autres objectifs des politiques éducatives comme la promotion de l'égalité des chances et peut ouvrir la voie -là n'est pas le moindre des paradoxesà la demande de moyens supplémentaires par les professionnels travaillant dans les quartiers populaires ou en charge des publics particulièrement défavorisés.

Les comparaisons internationales et le courant de recherche inspiré par l'efficacité de l'écoles apparaissent aujourd'hui comme ayant participé à des modifications 
profondes des systèmes d'éducation/formation. Parfois à leur corps défendant (Mons 2008), elles ont légitimé des politiques publiques qui les ont utilisées pour transformer les cadres du travail pédagogique en renvoyant d'abord la responsabilisation de l'action éducative aux personnels et à leur exercice dans des établissements de plus en plus autonomes (Dutercq \& Maroy 2017).

\section{De la mise en ouvre des réformes aux usages et pratiques professionnelles}

Le sens (la signification et les directions) de l'introduction de la NGP en éducation n'est donc pas univoque. Il compte des zones d'ambivalence, voire de contradictions. Ces dernières sont vives pour les personnels enseignants, en raison de la difficile codification de savoir-faire de métiers qui incluent nécessairement une action discrétionnaire des professionnels de base (Bezes et al. 2011), leur prodiguant des marges d'autonomie (Siggen 2015) variables pour répondre aux demandes des usagers.

Si la reconfiguration de l'action de l'État dans le domaine éducatif peut apparaître comme le vecteur de l'affirmation d'une gouvernance par le haut des pratiques enseignantes et de leurs effets, elle s'incarne au travers de ses mises en œuvre dans les établissements, créant l'effet baroque relevé par Demazières et al. (2013). Il l'est d'autant plus en éducation et formation qu'à l'usage du pilotage par les résultats s'ajoute une exigence élevée du maintien de pratiques prudentielles. La reddition de compte, la redevabilité, au cœur de la NGP, offre ainsi des possibilités d'action peu compatibles avec le contrôle managérial et la standardisation des instruments sur lesquels il repose, en partie au moins.

L'exigence de responsabilisation, faite aux personnels par la NGP, est en plus elle-même porteuse d'ambivalences, voire de contradictions. Ettre responsable face aux objectifs fixés nationalement ou localement demande en effet d'endosser leur réalisation comme partie intégrante de l'activité professionnelle, et ceci même ad minima. Or la conflictualité demeure pour les politiques éducatives dans plusieurs pays d'Europe (la tension entre valeurs marchandes et valeurs civiques marque certaines de ces tensions, mais les conflits ne s'arrêtent pas là et ils touchent parfois aussi l'organisation elle-même).

Aussi Moachon (2015, 53 et svts) remarque-t-il qu'un "florilège" de contradictions existe dans la mise en œuvre de la NGP et qu'il va bien au-delà de ses trois modèles idéal-typiques : l'efficience qui s'inspire du marché; la flexibilité et la décentralisation qui sont au cœur de la réduction des effectifs; la qualité qui prend l'excellence comme base. Il note ainsi, à propos des professionnels de l'insertion socio-professionnelle, que leur action est marquée par de multiples tensions en sus de la confrontation des valeurs marchandes aux valeurs civiques dans la recherche d'efficience. Elles proviennent des contradictions qui organisent le difficile équilibre entre autonomie et contrôle des professionnels par leurs hiérarchies ainsi que de la multiplicité des figures à qui il faut rendre des comptes. Le forum privilégié est-il celui des parents d'élèves? de la hiérarchie de proximité? des pairs syndiqués? des enseignants d'une même discipline? de l'État, en tant que garant des valeurs civiques ou qu'employeur?

Au-delà des principes de cette nouvelle donne, il s'agit de prendre en compte les traditions nationales et leurs histoires (Derouet \& Normand 2016 pour la France, Levasseur 2015 pour le Québec) et surtout les configurations locales et la force de résistance (d'adhésion ou de résilience) des groupes professionnels. Les remaniements en cours n'ont pu qu'influer sur les représentations que les personnels éducatifs ont de leur travail, des collaborations qu'ils et elles doivent nouer et de leur place dans des systèmes complexes. Ces formes de coordination sont loin d'aller de soi et aboutissent parfois à des concurrences marquées entre intervenants (Perez-Roux 2014), mais elles offrent aussi des marges à l'action dans certains segments professionnels, voire à des fractions de chacun d'eux.

La diversité des situations atteint ainsi les formes d'appropriation que les professionnels de différentes fractions du secteur public peuvent manifester. Elle est très marquée dans le système d'éducation/formation, les segments de ces professions enseignantes s'adressant à des publics distincts, ne jouissant pas de la même reconnaissance et travaillant dans des conditions très variées.

Parmi les enseignants, l'"ambivalence" vis-à-vis des réformes pourrait ainsi répondre à une hybridité organisationnelle, "résultante des tensions culturelles, identitaires et valorielles qu'immanquablement toute réforme contribue à raviver" (Emery \& Giauque 2014, 31), mais aussi résultante de trajectoires collectives et individuelles. Ces dernières ajustent les modalités de l'appropriation des réformes. Et ces "stratégies de valorisation" interne au groupe sont particulièrement saillantes dans les professions intermédiaires (infirmières ou enseignantes), qui ne disposent pas nécessairement du monopole sur un ensemble de savoirs et savoir-faire bien délimités 
(comme des groupes plus prestigieux : médecins avocats) (Morel 2014, 86-88).

\section{Pour une analyse socialement ancrée des "professionnalités" et de leurs métamorphoses}

\section{Un angle peu éclairé des analyses des réformes éducatives et de leurs effets}

En centrant la réflexion sur l'évaluation des effets des pratiques enseignantes et des modes de régulation des espaces professionnels de l'enseignement (établissements, académies...) sur les élèves et les apprentissages, les nombreuses études récentes sur les "professionnalité[s] enseignante[s] à l'heure des réformes" (Perez-Roux 2012) peuvent donner du personnel enseignant une vision assez désincarnée. Focalisées sur le contenu des réformes et leurs traductions en termes de formation et de modèles professionnels, elles minimisent les effets de l'inscription dans la structure sociale des personnes composant les groupes et fraction de groupes professionnels exerçant des activités d'enseignement quant à leurs pratiques et représentations.

Les enseignants et enseignantes de nombreux pays d'Europe et d'Amérique du Nord sont confrontés aujourd'hui à des changements importants semblant modelés par les mêmes logiques de "responsabilisation des enseignants' à l'égard de la réussite scolaire" (Dutercq \& Maroy 2017, 14). Ces réformes n'ont ni la même intensité ni la même forme selon les conjonctures politiques où elles sont appliquées (Mons \& Dupriez 2010), loin d'une poussée hégémonique d'un seul et même modèle, apparaît ainsi une "globalisation vernaculaire" des politiques d'éducation (Lingard 2006, Maroy, Pons \& Dupuy 2017) tant les cadres institutionnels et symboliques nationaux informent durablement leur mise en place et leurs effets concrets. Si ces nuances sont aujourd'hui plutôt bien connues, grâce aux travaux menés dans une perspective de "comparaison internationale", les variations intragroupes professionnels des effets de ces politiques restent assez peu analysées et, à de rares exceptions près (Farges, Guidi \& Métais 2018), peu problématisées dans les analyses des effets des réformes récentes sur les pratiques et les représentations des professionnels de l'enseignement. (cf. Ciringhuti ci-après).

Selon les capitaux sociaux et culturels dont elles et ils sont porteurs et porteuses, leurs modalités de formation et de sélection, leur socialisation genrée et tout ce qui structure leur cheminement de vie au sens large, leurs manières de s'approprier, de négocier, voire de contester ces injonctions professionnelles ambivalentes, varient nécessairement. Du fait de leur parcours scolaire ou social, des fractions de groupes professionnels tendent à être plus motrices dans la diffusion des pratiques managériales car elles peuvent en tirer profit quant à leur autonomie relative et à leur degré de responsabilité. C'est le cas, dans l'espace professionnel infirmier suisse, des membres de "l'élite infirmière", plus dotés en capitaux scolaires et plus jeunes que la majorité de leurs collègues (Longchamp et al. 2018, 240-241). La sociologie des organisations et de l'éducation montre par ailleurs que de telles mesures ne s'imposent pas uniquement de l'extérieur aux professionnels : "les changements impulsés ne peuvent s'opérer sans la participation active d'une partie des professionnels concernés qui ont ainsi l'occasion de transformer leur position au sein d'un espace de concurrence" (Garcia \& Montagne 2011, 11). Pour d'autres fractions, les réformes inspirées du NPM constituent une réduction de leur marge d'autonomie et affecte la construction de leur "identité au travail" (Hughes 1996, 75-85).

Le risque de dé-professionalisation que font planer les politiques de responsabilisation (reddition de compte, évaluation des performances des personnels enseignants) - pointées par ailleurs par des recherches depuis de nombreuses années (Cattonar \& Maroy 2000) - n'affecte-t-il pas en priorité certaines catégories de personnel porteuses de types de capitaux particuliers, de visions de la carrière et de certains rapports au travail? De la même façon, il faut s'interroger sur le fait que les mêmes politiques tendent au contraire à renforcer le sentiment de professionnalité chez d'autres fractions de personnels, s'opposant aux premières dans l'espace professionnel -comme pour les membres de l'élite infirmière romande précitée. C'est peut-être un des effets collatéraux les plus manifestes de la mise en place des injonctions à la reddition de compte. Ces nouvelles manières de gérer ces espaces professionnels du service public auraient ainsi pour conséquence minimale de révéler les fractures sociales internes de ces groupes professionnels (Bellini \& Maestripieri 2018). Dans l'espace enseignant, elles pourraient contribuer à amplifier les distances entre les différents segments professionnels, voire même favoriser des phénomènes reproduisant les stratifications sociales actuelles. C'est en tout cas l'hypothèse formulée ici à la lumière des différents articles qui composent ce dossier. 


\section{Féminisation et changement du niveau scolaire et social de recrutement : éléments de morphologie sociale des espaces enseignants}

Cette perspective vise à considérer le travail comme "comme un espace supplémentaire de l'expression des divisions sociales qui structurent les sociétés contemporaines" (Quijoux 2015, 55). Elle nécessite la mobilisation d" une approche structurale" (Pichonnaz \& Toffel 2018, 8) des professions enseignantes dont le but serait de replacer les professionnels en question au sein des rapports sociaux qui traversent leurs espaces sociaux spécifiques dans les sociétés où ils s'inscrivent et de "réencastrer" les professionnalités dans les parcours de vie des enseignants (Quijoux \& Pagis 2019, 8).

Les travaux de Merton (1957, 316-324) sur la "socialisation anticipatrice" ont montré qu'au moment d'entrer dans le métier, les individus sont "porteurs de dispositions qui sont déterminantes sur leurs pratiques et qui, comme elles sont incorporées, ne peuvent pas être détruites ou inhibées sans un travail ou un contexte particuliers" (Darmon 2018, 118). Ces dispositions orientent donc les appréhensions et les appropriations du métier. Mais de possibles désajustements entre les attentes (structurées par les produits des socialisations antérieures ou parallèles à cette entrée) et la réalité vécue peuvent ensuite engendrer des sorties de route ou des stratégies de contournement qu'il s'agit aussi de situer socialement, tant elles informent sur les dynamiques en cours au sein de l'espace professionnel étudié (Bataille 2017). Cette approche visant la "mise en espace social" (Darmon 2008, 152) des évolutions des professionnalités et des pratiques concrètes implique de porter le regard sur deux dimensions trop souvent négligées dans la sociologie de groupes professionnels : les dynamiques des modes d'accès aux espaces professionnels et de leur recrutement social premièrement; les modes de sortie ou de contournement parallèle qu'ils mettent en place deuxièmement.

Cette dimension, portant sur les possibles reconversions ou les contournements, est au cœur de plusieurs articles qui pointent combien les marginalisations -et les souffrances qu'elles peuvent engendrer- et les bifurcations sont socialement situées (cf. ci-après Garcia et al. et Devineau). Elle met également au jour les manières dont les réformes gestionnaires contribuent à rendre évidentes la division sociale du travail au sein des établissement scolaires et les polarisations professionnelles qui lui préexistent (Spire 2010 et ciaprès Vivier et al.).
La dimension assez classique, relative à l'analyse de l'effet du recrutement social des groupes professionnels sur les dynamiques professionnelles est assez peu traitée dans les travaux réunis dans ce dossier. C'est pourquoi ce texte introductif rappelle quelques éléments saillants quant à l'évolution de la morphologie sociale du corps enseignant depuis une trentaine d'années.

Trois points sont ici à souligner sur les métamorphoses de la plupart des professions enseignantes dans les différents degrés traités par les articles de ce dossier (primaire et secondaire) en Europe occidentale et en en Amérique du Nord : l'augmentation progressive, continue mais inégale, de la proportion des femmes dans l'ensemble des segments professionnels du monde enseignant, l'accroissement du niveau scolaire de recrutement des personnels et les changements induits sur leur recrutement social.

La féminisation de métiers de l'enseignement (surtout en primaire et secondaire) est un phénomène connu qui a touché les quatre aires nationales traitées (Belgique, Canada, France et Suisse). Les données disponibles (OCDE 2018) montrent ainsi que dans les générations les plus anciennes, la part de femmes enseignantes oscille entre 30 et $40 \%$, alors qu'elle dépasse systématiquement les $50 \%$ pour les plus récentes, au secondaire ou au primaire. Les conséquences de cette transformation structurelle de la profession ont fait l'objet de nombreux travaux (Cacouault-Bitaud 2007, Jarty 2014, Fassa \& Dubois 2017, Letrait \& Salane 2015, Moreau 2011) sur le rapport au travail des enseignants et enseignantes. Il est impossible de résumer ici leurs conclusions, tant les environnements investigués et les dimensions d'analyse mobilisées sont nombreux. Un résultat prévaut pourtant : si les carrières enseignantes du primaire et du secondaire ont pu être visées par certaines femmes à partir des années 1980 parce qu'elles paraissaient plus à même de permettre une conciliation entre vie de famille et vie professionnelle, l'analyse de l'expérience concrète des professionnelles révèle qu'elle est loin d'être aussi fluide que prévue, s'apparentant souvent même à un jonglage (Fassa 2016 en ligne). Du fait de configurations conjugales asymétriques où les enseignantes se trouvent souvent, la féminisation des métiers de l'enseignement entraîne in fine pour des parts croissantes du personnel des situations d'intensification du temps de travail et de flou des frontières entre les sphères d'activité privées et professionnelles. Au vu des débats concernant les effets de la NGP sur l'égalité des chances pour les élèves, la mise en place de la NGP mérite interrogation : ne jouet-elle pas aussi un rôle dans la reproduction sociale 
et genrée (Fassa, Benninghoff \& Kradolfer 2019) pour les professionnels, concourant à révéler et amplifier des fractures déjà existantes entre fractions des mondes de l'éducation et de la formation (Fassa \& Dubois 2019).

L'augmentation du niveau scolaire de recrutement est aussi connue. Elle apparaît comme est une conséquence mécanique de l'universitarisation de la formation des enseignants -soit "le processus qui [...] rend universitaires [les formations enseignantes] alors qu'elles existaient auparavant ailleurs qu'à l'université" (Bourdoncle 2007)- qui s'opère dans la plupart des pays européens et nord-américains depuis les années 1960 (pour le Québec, Lessard \& d'Arrisso 2010; pour une comparaison des conséquences de l'universitarisation de la formation enseignante en Europe, Bourdoncle 1997).

L'évolution du recrutement social en revanche reste une dimension de la morphologie sociale des différents corps enseignants moins analysée et plus sujette à controverses. Le cas du primaire français notamment a donné lieu à des analyses contradictoires (Vallet \& Degenne 2000, Charles \& Cibois 2010), même en considérant que l'origine sociale des "enseignants du premier degré s'est globalement élevée" (Farges 2011, 171). La "moyennisation" du recrutement des enseignants du secondaire à partir de la fin des années 1970 est assez établie en France (Chapoulie 1987, Farges 2011). Les analyses équivalentes ailleurs sont trop rares pour comparer les effets de l'universitarisation de la formation des enseignants sur leur recrutement social - parfois vu comme une des clés de compréhension des crises des espaces enseignants contemporains (Dubet 2014). Ce manque indique une possible orientation pour des recherches futures, qui viendraient utilement compléter les analyses des politiques publiques de la formation enseignante.

\section{Présentation des contributions}

Les contributions réunies dans ce dossier démontrent la nécessité de recourir à l'étude de cas élargie (Burawoy 2003) pour comprendre ce que font les principes de la NGP aux fractions des professionnels de terrain en éducation. Analysant la situation dans quatre pays ou régions francophones, elles mettent en évidence des réalités particulières, toutes débitrices d'un travail situé de réinterprétation et de reformulation lors de leur (parfois toute relative) mise en œuvre. N'en déplaise aux tenants d'une approche globale (Flitner 2012), elles insistent toutes sur le fait qu'une appréciation sérieuse des effets de la NGP sur les mondes de l'éducation/formation et sur leurs personnels - notamment sur les définitions de la professionnalité, le travail bien fait du monde anglo-saxon-doit prendre en compte les sociohistoires de chacun des espaces territoriaux, professionnels et politiques analysés et des chemins de dépendance (path dependancy, Pierson 1994) tracés par les choix antérieurs.

En éloignant le débat du conflit principiel et de ses références anglo-saxonnes, la focale adoptée permet une étude plus approfondie des recompositions en cours et invite à examiner ce que la NGP fait aux professionnels, mais aussi ce que les différents groupes professionnels font de la NGP.

Comme le relevaient Demazières et al. (2013), ces réformes ont des conséquences variables en termes de gain et de perte d'autonomie et de reconnaissance, car les professionnels de l'enseignement sont loin de constituer un groupe unique, homogène et soudé par une communauté d'intérêts et de valeurs. Au contraire, au-delà de leurs appartenances à différents segments professionnels existants ou potentiellement émergeants, ils s'adaptent ou rompent face aux mutations de leur environnement professionnel selon des configurations qui mettent en jeu l'ordre local, les appartenances syndicales pouvant par ailleurs accentuer les fractures entre les membres des hiérarchies intermédiaires et ceux qui pratiquent avant tout un métier de contact direct avec les usagers.

Les contributions réunies ici examinent, à partir de recherches de terrain, comment se construisent les dialectiques complexes alliant NGP, réformes inspirées par le courant de la recherche et usages différenciés que les groupes d'acteurs et actrices font de cette nouvelle donne. Elles donnent aussi à voir un fort degré d'hybridation entre diverses conceptions de la professionnalité et des groupes d'acteurs et d'actrices qui tendent à maintenir et/ou améliorer leur situation professionnelle tout en répondant à des missions de service public, dont les apprentissages et la réussite des enfants et des jeunes et qui s'actualisent toujours dans des ordres locaux spécifiques.

S'inscrivant dans l'approche cognitive des politiques publiques, la contribution de Branka Cattonar et Vincent Dupriez ouvre le dossier. Elle met en lumière la tension entre deux modes de professionnalité proposés par un même texte qui veut servir de référentiel au pilotage de la réforme dans la Fédération WallonieBruxelles. Produit par un groupe incluant toutes les catégories d'acteurs et d'actrices, il a donné lieu à de nombreux compromis et laisse voir une lecture très hybride de ce que devrait être le modèle idéal de 
professionnalité enseignante . Il articule un modèle qui peut renvoyer à celui à du praticien réflexif, engagé par une collaboration participative dans son environnement professionnel, mais cadré par une obligation commune faite à l'ensemble des segments professionnels d'adopter les impératifs de la reddition de comptes et de la responsabilisation à l'égard des résultats (cf. le professionnalisme managérial, Demailly 1998). La description des attentes en termes de professionnalité enseignante donne une image très parlante de l'hybridation en cours. Ici, elle mène à un modèle qui repose à la fois sur une forme de hiérarchisation horizontale (avec pour horizon la professionnalisation et la collégialité) qui accepterait néanmoins les référentiels des politiques publiques de la NGP comme guides pour l'action.

L'article d'Hélène Buisson-Fenet et Xavier Pons insiste sur les disparités existantes des modes d'appropriation de la NGP en examinant les modalités du "découplage" (Bezès et al. 2011, 310) entre les propositions de réformes et les mises en œuvre dans six lycées français. Étudiant les discours des acteurs professionnels, il montre que les effets des réformes et leur adoption s'avèrent fondamentalement différents selon les rapports établis entre les groupes professionnels de chaque établissement et les manières dont ils conçoivent leur professionnalité. Ce faisant, il insiste sur la nécessité d'un examen très approfondi des situations et conclut à l'importance de l"'ordre local" lorsqu'il s'agit de débattre des réformes de la NGP et l'acceptation de ses instruments. Bien qu'elle ne le dise pas explicitement, cette contribution met aussi l'accent sur le fait que la reconfiguration de l'action publique liée à la NGP ne profite pas identiquement à l'ensemble des acteurs et actrices. Les lycées dans lesquels la collaboration semble prévaloir pour l'implémentation des instruments proposés sont aussi ceux qui bénéficient d'une certaine reconnaissance du fait de leur mission particulière ou des publics qui les fréquentent.

La contribution de Simon Viviers, Anne Abdoulaye et Patricia Dionne (ci-après) poursuit dans la même veine. S'intéressant à l'évolution du métier des Conseillers en orientation au Québec, elle décrit finement cinq profils d'appropriation des changements induits par la volonté des autorités de les rendre plus polyvalents en orientant aussi leurs activités vers l'intervention clinique auprès d'élèves dits vulnérables. Elle montre combien l'incidence des politiques de reddition de compte sur les pratiques professionnelles ainsi que leur possible traduction en facteur aggravant la souffrance au travail varie fortement selon l'environnement. Entre professionnels au cœur de l'espace professionnel du conseil en orientation et ceux plus marginaux (ayant des contrats plus précaires ou œuvrant davantage dans la formation professionnelle), la tension autour de l'identité de métier induite par les réformes inspirées de la NGP est radicalement différente. Selon les auteurs, ces réformes contribuent à mettre en souffrance les fractions de professionnels déjà peu reconnus et participent nettement d'une fracturation avancée du groupe professionnel en question.

La contribution de Laurent Frajerman (ci-après) thématise le fait que "tous ne gagnent pas également" à la mise en œuvre de l'action publique. Adoptant un point de vue de politiste et prenant en compte le positionnement syndical des différentes catégories professionnelles, l'auteur montre que les ambivalences qui ont marqué et marquent les réformes -et les atermoiements politiques qui les ont chapeautées (Derouet \& Normand 2016)- sont suffisamment fortes à tous les échelons pour que leur issue reste incertaine et dépende avant tout des manières dont les jeunes générations enseignantes, celles "qui n'ont pas été socialisées dans l'atmosphère antihiérarchique des années 1970", interagiront avec d'autres fractions du monde enseignant. Ce faisant, elle examine les jeux possibles autour des appartenances générationnelles et attire, elle aussi, l'attention sur une fracture inhérente aux trajectoires individuelles et aux situations où elles se sont déployées.

Les trois dernières contributions se focalisent sur les dynamiques des carrières enseignantes à deux moments cruciaux : l'entrée dans la carrière (Crispin Girinshuti ci-après) et les bifurcations hors du métier (Sandrine Garcia, Géraldine Farges, Magali Danner et Eloïse Fradkin; Sophie Devineau. Crispin Girinshuti analyse précisément, à partir du cas vaudois (Suisse), comment les enseignants débutants s'apparient avec leur établissement dans un moment de libéralisation du marché du travail enseignant local -où le recrutement est désormais a priori découplé de la formation. Il montre que, malgré cette tendance à la mise en marché de l'offre professionnelle enseignante, les logiques de la proximité géographique et de l'interconnaissance gardent une place prépondérante dans les appétences des néo-enseignants pour leur établissement. Ce n'est que dans un deuxième temps que la qualité de l'emploi et les conditions concrètes d'exercice (le temps de travail notamment) rentrent en ligne de compte. En écho avec des analyses classiques des carrières enseignantes (Becker 1952), la contribution rappelle que même à une époque où l'attractivité économique et matérielle des professions enseignantes est le principal levier politique pour susciter les vocations, 
les modalités d'orientation des nouveaux enseignants restent fortement structurées par des logiques plus socio-symboliques et interactionnelles qu'allant dans le sens d'une maximisation du profit tiré du capital scolaire individuel.

La contribution de Sandrine Garcia, Géraldine Farges, Magali Danner et Éloïse Fradkin et celle de Sophie Devineau ont en commun de préciser les usages que les professionnels font des réformes en examinant les sorties du métier. Se fondant sur les statistique ministérielles, les premières autrices montrent d'abord qu'elles sous-évaluent ce phénomène en ne prenant pas en compte les voies qui permettent de s'éloigner du travail dans la classe, "cœur du métier" enseignant. L'enquête menée dans une académie française met en évidence que les mises en disponibilité ou les détachements augmentent considérablement le nombre de personnes quittant l'enseignement primaire et secondaire. Elles pointent le fait que les éloignements du cœur du métier ont des caractéristiques locales qui renvoient aux effets des réformes comme motif de l'exit (Hirschman1973), mais aussi que les personnes ne sont pas égales pour effectuer des arbitrages face à une situation jugée intolérable pour une raison ou une autre. Elles soulignent ainsi que les issues des sorties de route dépendent grandement des ressources spécifiques dont disposent chaque personne.

Les ressources sont aussi au centre de l'analyse de Sophie Devineau, qui interroge ce qui fonde la reprise de formation des professeures des écoles expérimentées qui s'engagent dans "pilotage des dispositifs de prise en charge de la petite enfance". En resserrant encore la focale sur les personnes en conversion professionnelle, son autrice parvient à mettre en évidence au travers d'entretiens biographiques analysés dans une perspective de genre que ce type de trajectoire témoigne d'un "féminisme en acte" (Jacquemart \& Albenga 2015) qui répond au bousculement imposé par les réformes et le développement de dispositifs de gestion de l'éducation par une agentivité individuelle, socialement ancrée, faute de solutions collectives. Ces deux contributions saisissent ce que les réformes impliquent en termes de remaniements identitaires et elles montrent que les voies choisies varient en fonction des caractéristiques générationnelles (primo-enseignants ou non pour Garcia et al.), sociales (origine, situation maritale, capital scolaire et économique) et de genre des enseignants. Ces deux articles éclairent le fait que l'appropriation des réformes tient aussi aux particularités individuelles, tous et toutes n'y gagnant pas également.

La peinture livrée ici laisse percevoir que si la NGP marque les formes de carrière dans les diverses professions éducatives, leur mode de gestion ou les normes des différentes professionnalités, elle le fait de manière à chaque fois spécifique, ces distinctions se construisant au travers d'un jeu complexe qui inclut aussi les rapports de force entre des acteurs et des actrices ayant des caractéristiques sociodémographiques et des trajectoires différentes, des positionnements sociaux et politiques variés et vivant et travaillant dans des espaces sociaux et territoriaux particuliers, eux aussi parfois en conflit. Ces changements d'échelle (du global au local et du collectif aux individus) offrent une lecture qui permet de comprendre pourquoi les personnels de l'éducation et de la formation ne parviennent que difficilement à prendre des positions unifiées sur les nouveaux principes affirmés par la NGP. Les transformations en cours sont assez partagées pour ébranler les organisations professionnelles et les identités des personnels (privée mais surtout professionnelle) antérieures, mais aussi suffisamment distinctes pour que leurs réinterprétations soient marquées par des histoires, des traditions et des débats qui s'articulent à chaque fois de manière spécifique.

\section{Références bibliographiques}

BARRÈRE A. 2013 "La montée des dispositifs : un nouvel âge de l'organisation scolaire", Carrefours de l'éducation-36, 95-116

BATAILLE P.2017 "Des carrières ratées? Sociogenèses et appropriations d'une bifurcation : le cas des anciens élèves des ENS enseignants dans le secondaire", Biens Symboliques/Symbolic Goods-1, en ligne $<$ http://revue.biens-symboliques.net/ $112>$, le $22-08-2019$

BAUDELOT C. \& ESTABLET R. 2009 L'élitisme républicain, Paris, Seuil

BECKER H. 1952 "The career of the Chicago public schoolteacher", American Journal of Sociology$57,470-477$

BEZES P., DEMAZIÈRE D. \& LE BIANIC T. 2011 "New Public Management et professions dans l'État : au-delà des oppositions, quelles recompositions?" Sociologie Du Travail, 53(3), 293-348

BELLINI A. \& MAESTRIPIERI L. 2018 "Professions Within, Between and Beyond. Varieties of Professionalism in a Globalising World", 8(16), 5-14

BOURDONCLE R.1997 "Normalisation, académisation, universitarisation, partenariat : de la diversité des voies vers l'université", Revista da Faculdade de Educação-23(1-2), en ligne <https://dx.doi.org/ 
10.1590/S0102-25551997000100004> consulté le 2208-2019

BOURDONCLE R. 2007, "Universitarisation" , Recherche formation-54, 137-149

BRUNO I., CLÉMENT P. \& LAVAL C. 2011 La grande mutation. Néolibéralisme et éducation en Europe Paris. Syllepses

BRESSOUX P. 2009 Des contextes scolaires inégaux : effet-établissement, effet-classe et effets du groupe de pairs, in Duru-Bellat M. \& van Zanten A. éds. Sociologie du système éducatif. Les inégalités scolaires, Paris, PUF, 131-148

BURAWOY M. 2003 "L'étude de cas élargie. Une approche réflexive, historique et comparée de l'enquête de terrain", in CÉFAÏ D. dir. L'enquête de terrain, Paris, La Découverte/M.A.U.S.S, 425-464

CACOUAULT-BITAUD M. 2007 Professeurs... mais femmes. Carrières et vies privées des enseignantes du secondaire au $X X^{e}$ siècle, Paris, La Découverte

CATTONAR B. 2001 "Les identités professionnelles enseignantes. Ébauche d'un cadre d'analyse", Cahier de Recherche Du GIRSEF-10, 1-35

CATTONAR B. \& MAROY C. 2000 "Rhétorique du changement du métier d'enseignant et stratégie de transformation de l'institution scolaire", Éducation et Sociétés-6, 21-42

CHAPOULIE J.-M. 1987 Les professeurs de l'enseignement secondaire : un métier de classe moyenne, Paris, Éditions de la MSH

CHARLES F. \& CIBOIS P. 2010 "L'évolution de l'origine sociale des enseignants du primaire sur la longue durée : retour sur une question controversée", Sociétés contemporaines-77, 31-55

CE (Commission européenne) 2012 Communication de la commission au parlement européen, au conseil, au comité économique et social européen et au comité des régions. Repenser l'éducation-Investir dans les compétences pour de meilleurs résultats socioéconomiques, Strasbourg

DARMON M. 2008 "La notion de carrière : un instrument interactionniste d'objectivation", Politix82, 149-167

DARMON M. 2018 "La socialisation secondaire ne s'exerce pas sur une page blanche mais sur une page déjà écrite et déjà froissée par les expériences antérieures", Émulations -Revue de sciences sociales25, 115-121

DEMAILLY L. 1998 "Les métiers relationnels de service public : approche gestionnaire, approche politique", Lien social et Politiques-40, 17-24

DEMAZIĖRES D., LESSARD C. \& MORRISSETTE J. 2013 "Introduction. Les effets de la Nouvelle
Gestion Publique sur le travail des professionnels : transpositions, variations, ambivalences". Éducation et Sociétés-32, 5-20

DEROUET J.-L. \& NORMAND R. 2016 New Public Management in the french educational system between affirmation of the State and decentralized governance, in Gunter H., Grimaldi E, Hall D. \& Serpieri R. New Public Management and the reform of education. European lessons for policy and practice, London-New York, Routledge

DUBET F. 2009 "Penser les inégalités scolaires", in Duru-bellat M. \& van Zanten A. dirs. Sociologie du système éducatif. Les inégalités scolaires, Paris, PUF, $17-34$

DUBET F. 2014 Les places et les chances : repenser la justice sociale, Paris, Seuil

DUBET F., DURU-BELLAT M. \& POULLAOUEC T. 2006 "Polémiques sur l'utilité des diplômes", Revue du MAUSS-28(2), 85-95.

DUBET F., DURU-BELLAT M. \& VÉRÉTOUT A. 2011 "Emprise des diplômes, jugements de justice et cohésion sociale". Sociologie et Sociétés-43(1), 225-259

DURU-BELLAT M. 2011 Quelle marge de manœuvre pour l'école dans un environnement d'inégalités? in Paugam S. éd. Repenser la solidarité : L'apport des sciences sociales, Paris, PUF, 669-685

DUTERCQ Y. \& MAROY C. 2017 "Introduction", in Dutercq Y. \& Maroy C. dirs. Le professionnalisme enseignant face aux politiques de responsabilisation, Louvain-la-Neuve, De Boeck Supérieur

EMERY Y. \& GIAUQUE D. 2014 "L'univers hybride de l'administration au XXI ${ }^{\mathrm{e}}$ siècle". Revue Internationale Des Sciences Administratives-80(1), 25-34

FARGES G. 2011 "Le statut social des enseignants français", Revue européenne des sciences sociales49(1), 157-178

FARGES G., GUIDI P. \& MÉTAIS J. 2018 "Introduction. Saisir les transformations des conditions enseignantes dans leur diversité et leur complexité", Cahiers de la recherche sur l'éducation et les savoirs17, 7-20

FASSA F. 2016 "Articulation des temporalités et régimes de genre : qui gagne quoi[202F?]?" SociologieS, (October), 0-14, en ligne <https ://sociologies.revues.org/5779>

FASSA F. \& DUBOIS S. 2019 "Les enseignants de la formation professionnelle initiale : parcours professionnels et rapports aux savoirs à transmettre", dans Perez-Roux T., Deltand M., Duchesne C. \& Masdonati J. dir. Parcours, transitions professionnelles et constructions identitaires : le sujet au cœur des 
transformations, Montpellier, Presses universitaires de la Méditerranée, 221-241

FASSA F., BENNINGHOFF M. \& KRADOLFER S. 2019 sous presse, "Universités : les politiques d'égalité entre femmes et hommes à l'heure de l'excellence" introduction, SociologieS

FASSA F. \& DUBOIS S. 2017 "À l'heure des incertitudes : qui résiste à la démocratisation de l'accès aux études supérieures", Spirales-60, 71-87

FELOUZIS G. \& CHARMILLOT S. 2012. Les enquêtes PISA, Paris, Presses universitaires de France

FLITNER E. 2012 "World polity -Théories et recherches sur la mondialisation des politiques éducatives", Recherche et formation-69, 101-110

GARCIA S. \& MONTAGNE S. 2011 "Pour une sociologie critique des dispositifs d'évaluation", Actes de la recherche en sciences sociales-189, 4-15

HIRSCHMAN A.O. 1973 "'Exit, voice and loyalty' : Further reflections and a survey of recent contributions". Social Sciences Information-13(1), 7-26

HUGHES E.C. 1996 Le regard sociologique, Paris, Éditions de l'EHESS

JACQUEMART A. \& ALBENGA V. 2015 "Pour une approche microsociologique des idées politiques. Les appropriations ordinaires des idées féministes", Politix-109(1), 7-20

JARTY J. 2014 "Les usages de la flexibilité temporelle chez les enseignantes du secondaire", Temporalités-9, en ligne <https://journals. openedition.org/temporalites/1057> consulté le 22-08-2019

LANG V. 2001 "Les rhétoriques de la professionnalisation", Recherche et formation-38, 95-117

LAVAL C. 2003 L'école n'est pas une entreprise. Le néo-libéralisme à l'assaut de l'enseignement public, Paris, La Découverte

LAVAL C, VERGNE F., CLÉMENT P. \& DREUX G. 2011. La nouvelle école capitaliste, Paris, La Découverte

LETRAIT M. \& SALANE F. 2015 "Le temps consacré aux enfants : les enseignantes et enseignants se distinguent-ils des autres diplômés de l'enseignement supérieur?", Économie et statistique-478(1), 243-271

LESSARD C. \& D'ARRISSO D. 2010 'L'universitarisation de la formation des enseignants. Exemple du Québec". Recherche et formation-65, 31-44

LEVASSEUR L. 2015 "Quebec's bifurcated schools : Tensions between democratic and commercial values", European Educational Research Journal-14(3-4), 364376

LINGARD B. 2006 "Globalisation, the research imagination and deparochialising the study of education", Globalisation, Societies and Education4(2), 287-302

LONGCHAMP P., TAWFIK A., BÜHLMANN F. \& TOFFEL K. 2018 "L'espace professionnel infirmier : une analyse à partir du cas de la Suisse romande", Revue française de sociologie-59(2), 219-258

MAROY C. 2006 "Les évolutions du travail enseignant en France et en Europe[202F ?] : facteurs de changement, incidences et résistances dans l'enseignement secondaire", Revue Française de Pédagogie-155, 111-142

MAROY C 2008 "Perte d'attractivité du métier et malaise enseignant", Recherche et formation-57, 23-38

MAROY C. 2018 "Nouvelles figures du 'social' et reconfigurations de la normativité scolaire", Raisons Éducatives-22, 277-294

MAROY C., PONS X. \& DUPUY C. 2017 "Vernacular Globalisations : Neo-Statist Accountability Policies in France and Quebec Education", Journal of Education Policy-32(1), 100-122

MERTON R.K. 1957 Social theory and social structure, New York, Free Press Of Glencoe

MOACHON E. 2015 Impact des instruments de redevabilité sur la mise en œuvre des politiques d'insertion socioprofessionnelle en Suisse romande. Thèse de doctorat en Science politique, Université de Lausanne

MONS N. 2008a "Évaluation des politiques éducatives et comparaisons internationales", Revue Française de Pédagogie-164(5), 5-13

MONS N. 2008b "Élites scolaires, inégalités sociales et renouveau des filières dans l'École moyenne[202F ?] : une comparaison internationale", Éducation et Sociétés-21, 17-32

MONS N. \& DUPRIEZ V. 2010 "Les politiques d'accountability. Responsabilisation et formation continue des enseignants", Recherche et formation-65, 45-59

MOREAU M.-P. 2011 Les Enseignants et le Genre, Paris, PUF

MOREL S. 2014 "Catégories de l'entendement et modes de valorisation professionnelle chez les formateurs de professeurs des écoles", Revue française de pédagogie. Recherches en éducation-189, 77-89

NORMAND R. \& DEROUET J.-L. 2011"Évaluation, développement professionnel et organisation scolaire", Revue française de pédagogie,174, p. 5-20.

OCDE (Organisation de Coopération et de développement économique) 2004 Apprendre aujourd'hui, réussir demain, rendant compte des premiers résultats de l'enquête PISA 2003

OCDE (Organisation de Coopération et de développement économique) 2018 Regards sur l'éducation 2018 : Les indicateurs de l'OCDE, Paris, OCDE 
PEREZ-ROUX T. 2012 "Construire une professionnalité enseignante à l'heure des réformes : enjeux deet défis pour- la formation", dans Perez-Roux T. dir. Rennes, PUR, 97-120

PEREZ-ROUX T. 2014 "Dynamiques identitaires d'acteurs de l'éducation en période de transition", Sociologies pratiques-28, 41-52

PICHONNAZ D. \& TOFFEL K. 2018 "Pour une analyse dispositionnelle des pratiques professionnelles", Émulations-Revue de sciences sociales-25, 7-21

PIERSON P. 1994 Dismantling the Welfare State? Reagan, Thatcher, and the Politics of Retrenchment. Cambridge, Cambridge University Press

POULLAOUEC T. 2010 Le diplôme, arme des faibles. Les familles ouvrières et l'école, Paris, La Dispute

QUIJOUX M. 2015 "La fabrique du travailleur : reproduction sociale, habitus et champ", in Quijoux M. dir. Bourdieu et le travail, Rennes, PUR, 41-63

QUIJOUX M. \& PAGIS J. 2019 "Des ressorts aux incidences biographiques du travail", Terrains travaux34(1), $5-18$

SIGGEN M. 2015 Régulation professionnelle dans un contexte de changement. Le cas des enseignantschercheurs à l'Université de Lausanne. Thèse de doctorat ès Science politique. Université de Lausanne

SPIRE A. 2010 "Les effets politiques des transformations du corps enseignant", Revue française de pédagogie. Recherches en éducation-170, 61-72

VALLET L.-A. \& DEGENNE A. 2000 "L'origine sociale des enseignants par sexe et niveau d'enseignement", Éducation et formation-56, 33-40 